\title{
CORRESPONDENCES CONNECTED WITH A PENCIL OF $n$-ICS*
}

\author{
BY A. R. WILLIAMS
}

1. Introduction. All curves of order $n$ passing through $(n / 2)(n+3)-1$ points pass through $(n-1)(n-2) / 2$ other points. That is, the $n^{2}$ base points of a pencil of $n$-ics are determined by the former number. When $(n / 2)(n+3)-2$ are fixed in general position, and another moves on a curve of order $m$, the locus of the remaining $(n-1)(n-2) / 2$ is a curve of order $m\left(n^{2}-1\right)$, which has a multiple point of order $m n$ at each of the fixed points. $\dagger$ In a previous paper I have considered the case when a number of base points are fixed and the others necessary to determine a pencil are taken consecutive on some curve. $\ddagger$ One other situation is perhaps worth brief notice. Of the $n q$ intersections of two curves of order $n$ and $q$, $n>q, n q-(q-1)(q-2) / 2$ can be taken arbitrarily, the others being then determined. Moreover,

$n q-\frac{(q-1)(q-2)}{2}+\frac{(n-q)(n-q+3)}{2}-1=\frac{n(n+3)}{2}-2$.

This means that if $n q-(q-1)(q-2) / 2$ base points are taken on a curve of order $q$, and $(1 / 2)(n-q)(n-q+3)-1$ others not on the latter, then if one other, say $P$, describes some locus, $(q-1)(q-2) / 2$ will be fixed on the curve of order $q$, and the remaining $(1 / 2)(n-1)(n-2)-(1 / 2)(q-1)(q-2)$ $=(1 / 2)(n-q)(n+q-3)$, variable with $P$, will, for any position of $P$, lie on the curve of order $n-q$ determined by $P$ and the second set of fixed points just mentioned. It is the purpose of this paper to discuss the locus of the variable points when $P$ describes a given curve.

2. Order of the Locus and Its Singularities at the Base Points. In this connection we may use the rational surface whose plane

* Presented to the Society, April 6, 1935.

$\dagger$ Milinowski, Journal für Mathematik, vol. 77, p. 263.

$\ddagger$ Williams, this Bulletin, vol. 36, p. 133. 
sections correspond to the $\infty^{3} n$-ics that have in common the $n q$ fixed points on the $q$-ic and all but one of the others. It will be convenient to refer to the set on the $q$-ic as the points $A$, and the others, $(1 / 2)(n-q)(n-q+3)-2$ in number, as the points $B$. The remaining fixed point, which reduces the multiplicity of $n$-ics to a net, may be called $Q$. The points $B$ and $Q$ determine a pencil of $(n-q)$-ics. The order of the surface is $(1 / 2)(n-q)(n+q-3)+2$. It has a multiple point of order $q(n-q)$ corresponding to the $q$-ic. The sections through the multiple point correspond to the net of $(n-q)$-ics determined by the points $B$. In particular, to the points $A$ on the $q$-ic correspond $n q$ lines of the surface which pass through the multiple point. To a pencil of $n$-ics determined by the points $A, B, Q$, and $P$ corresponds a pencil of plane sections whose axis is $Q^{\prime} P^{\prime}$, the latter being the points of the surface that correspond to $Q$ and $P$. The axis $Q^{\prime} P^{\prime}$ meets the surface in $(1 / 2)(n-q)(n+q-3)$ other points to which correspond in the plane the remaining base points of the pencil of $n$-ics. As $P$ moves on a general line $l, P^{\prime}$ moves on an $n$-ic, which is skew unless $q=n-1$, and has a multiple point of order $q$ at the multiple point of the surface. Thus the cone generated by $Q^{\prime} P^{\prime}$ has a $q$-fold edge. It meets the surface in the skew $n$-ic and a curve whose image in the plane is the locus sought. To find the order of the latter we have only to note that to a second line $l^{\prime}$ of the plane corresponds a similar $n$-ic on the surface which meets the cone described by $Q^{\prime} P^{\prime}$ in $n^{2}$ points, $q^{2}$ of which are at the multiple point of the surface, while another corresponds to the intersection of $l$ and $l^{\prime}$. Hence as $P$ moves on $l$, the variable points fill a curve $L$ of order $n^{2}-q^{2}-1$ which passes $n-q$ times through each of the points $A$, $n$ times through each of the points $B$, and $n$ times through the point $Q$, each passage through $Q$ corresponding to an intersection of the locus of $P^{\prime}$ with the tangent plane to the surface at $Q^{\prime}$. Thus, of the intersections of $L$ with the $q$-ic, $n q(n-q)$ are accounted for at the points $A$; and the remaining $q[q(n-q)-1]$ correspond to the $q$ intersections of $l$ with the $q$-ic. When $P$ takes a position on the $q$-ic, the pencil of $n$-ics becomes the $q$-ic and the pencil of $(n-q)$-ics determined by the points $B$ and $Q$. One of these passes through $P$ and meets the $q$-ic in $q(n-q)-1$ other points which correspond to $P$. The others are the $(1 / 2)(n-q-1)(n-q-2)$ remaining base points of the 
pencil of $(n-q)$-ics. The sum of the last two expressions is $(1 / 2)(n-q)(n+q-3)$, the number of points variable with $P$. The remaining base points of the pencil of $(n-q)$-ics correspond to the points in which the line joining $Q^{\prime}$ to the node of the surface meets the latter again. Each of them is a $q$-fold point on $L$, since $P$ in describing $l$ meets the $q$-ic $q$ times.

3. Self-Corresponding Points and Branch-Points. The locus of of points $P$ such that one of the corresponding points coincides with $P$ is the jacobian of the net of $n$-ics determined by the points $A, B$, and $Q$. It is the image of the curve of contact of the tangent cone drawn to the surface from $Q^{\prime}$. This jacobian contains the $q$-ic. The residual $J$, of order $3(n-1)-q$, meets $l$ in those points which are points of $L$. Of the other intersections of $J$ and $L, n-q$ are accounted for at each of the $n q$ points $A$, and $2 n$ at each of the points $B$ and at $Q$. This leaves $(n-q)\left(2 n^{2}+2 n q-6 n-3 q-q^{2}-2\right)-2(n-3)$ points of intersection which do not take place on $l$ or at any of the base points. These are points at which two of the points corresponding to a position of $P$ coincide. That is, they correspond to branch-points on $l$. In connection with the correspondence between the points of $l$ and $L$ we may apply the formula of Zeuthen, $\eta-\eta^{\prime}=2 \alpha\left(p^{\prime}-1\right)$ $-2 \alpha^{\prime}(p-1)$. Here $\alpha$ and $\alpha^{\prime}$ are 1 and $(n-q)(n+q-3) / 2$, while $\eta$ and $\eta^{\prime}$ are the numbers of branch-points on $l$ and $L$, and $p$ and $p^{\prime}$ are their genera. That is, $\eta$ is the number last determined, $\eta^{\prime}$ is 0 , and $p$ is 0 . Thus $p^{\prime}$, the genus of $L$, is $(\eta / 2)-\alpha^{\prime}+1$, which is found to be $(1 / 2)(n-q)\left(2 n^{2}+2 n q-q^{2}-4 q-7 n+1\right)-(n-4)$. It is of some interest to verify this number. The line $l$, the locus of $P$, and the corresponding locus $L$ meet in $n^{2}-q^{2}-1-3(n-1)+q$ or $(n-1)(n-2)-q(q-1)$ points which are not on $J$. These come in pairs, each pair corresponding to an apparent double point of the locus of $P^{\prime}$ as seen from $Q^{\prime}$. Moreover, with each such pair are associated $\alpha^{\prime}-1$ or $(1 / 2)(n-q)(n+q-3)-1$ nodes on $L$. For any one of the $\alpha^{\prime}+1$ associated points determines the others. Let $P_{1}$ be a point of $l$ for which one of the corresponding points, say $R_{1}$, falls on $l$. When $P$ describing $l$ reaches $R_{1}$, one corresponding point is at $P_{1}$ and the $\alpha^{\prime}-1$ others coincide with points corresponding to $P_{1}$, producing $\alpha^{\prime}-1$ nodes. Now the genus of $L$ found by considering the singularities at the points $A, B$, and $Q$, the $(1 / 2)(n-q-1)(n-q-2) q$-fold points common to the pencil 
of $(n-q)$-ics, and the $(1 / 2)[(n-1)(n-2)-q(q-1)]\left(\alpha^{\prime}-1\right)$ nodes just mentioned, agrees with the above value of $p^{\prime}$ given by the formula of Zeuthen.

If now $P$ moves on a curve $c$ of order $m$, the locus of the corresponding points is a curve $C$ of order $m\left(n^{2}-q^{2}-1\right)$ which has an $m n$-fold point at each of the points $B$ and at $Q$, and an $m(n-q)$-fold point at each of the points $A$. Each of the remaining base points of the pencil of $(n-q)$-ics is an $m q$-fold point of $C$. The number of branch-points on $c$ is also $m$ times the number on $l$. Zeuthen's relation then gives for the genus of $C, p^{\prime}=(\eta / 2)-\alpha^{\prime}+\alpha^{\prime} p+1$. Here $p$ is the genus of $c$, and $\eta$, the number of branch-points on $c$, is $m$ times the number of branchpoints on $l$. This shows, as is geometrically evident, that each node of $c$ gives $\alpha^{\prime}$ nodes on $C$. The curves $c$ and $C$ meet in $m\left(n^{2}-q^{2}-1\right)-m(3 n-3-q)$ points not on $J$. The last number may be written $(m n-1)(m n-2)-m q(m q-1)-(m-1)(m-2)$, which shows that it is twice the number of apparent double points of the locus of $P^{\prime}$. With each such apparent double point, or with each such pair of intersections of $c$ and $C$, are associated $\alpha^{\prime}-1$ nodes of $C$. Moreover, as just remarked, each of the $(1 / 2)(m-1)(m-2)-p$ nodes or cusps of $c$ gives $\alpha^{\prime}$ nodes or cusps on $C$. Then, considering the singularities of $C$ at the points $A, B$, and $Q$, the $m q$-fold points at the remaining base points of the pencil of $(n-q)$-ics, and the two classes of simple nodes just described, the genus of $C$ is found to agree with the above. It is easily shown that if the locus of $P$ passes through a point $B$ or through $Q$, the corresponding locus $C$ loses the $n$-ic of the net that has that point for double point. But if the locus of $P$ passes through a point $A$ on the $q$-ic, $C$ loses the $(n-q)$-ic that is determined by the points $B, Q$, and the point $A$ in question.

4. Particular Cases. The cases $q=n-1$ and $q=n-2$ are of interest. When $q=n-2$, there are only three points $B$. The number of base points variable with $P$ is $2 n-5$. If $P$ describes a line, the order of their locus is $4 n-5$. The order of the rational surface is $2 n-3$, and its multiple point is of order $2 n-4$, corresponding to the fact that the only base points of the pencil of conics are the three points $B$ and $Q$. The conics of the net determined by the points $B$ are the images of sections by planes through the multiple point. The double curve of the surface 
consists of three $(n-2)$-fold lines passing through the multiple point. Their images are the lines of the plane joining the points $B$ in pairs. That is, through the points $A$ and $B$ pass a web of $n$-ics, and the $n-2$ remaining intersections of an $n$-ic of this web with the line joining two points $B$ are determined by one of them. When $P$ moves on the line $B_{1} B_{2}$, the locus of the variable points is $B_{1} B_{2}$ taken $n-3$ times and $B_{3} Q$ taken $n-2$ times.

When $q=n-1$, there are no points $B$. When $Q$ is fixed, the number of points variable with $P$ is $n-2$. They lie on the line $Q P$; and if $P$ moves on a general line, their locus is of order $2(n-1)$, and has an $n$-fold point at $Q$ and simple points at the points $A$ on the $(n-1)$-ic. The rational surface is of order $n$, and has a multiple point of order $n-1$. Sections through the latter correspond to the lines of the plane. If $P$ describes a line passing through $Q$, the locus of the variable points is of course that line. But in this connection one fact may be noted. To the line joining $Q$ and $A_{k}$ on the $(n-1)$-ic corresponds on the surface a plane $(n-1)$-ic whose plane contains the line $a_{k}$ of the surface that corresponds to $A_{k}$. Hence, for any position of $P$ on the line $Q A_{k}$ one of the associated points is at $A_{k}$. That is, the pencil of $n$-ics have a common tangent at $A_{k}$, the one having a double point being the $(n-1)$-ic and the line.

Since the number of points $A$ on the $(n-1)$-ic is just sufficient to determine a web of $n$-ics, we may take $Q, P$ consecutive on some curve $c$ of order $m$. That question for the base points in general position was considered in the article referred to at the beginning of the paper. The modification to be made may be indicated briefly. To $c$ corresponds on the surface a curve $c^{\prime}$, of order $m n$, having an $m(n-1)$-fold point at the $(n-1)$-fold point of the surface. To a pencil of plane $n$-ics tangent to $c$ corresponds a pencil of plane sections whose axis is tangent to $c^{\prime}$ and meets the surface in $n-2$ other points, giving a curve on the surface whose image in the plane is the locus sought. The tangents to $c^{\prime}$ form a developable of order $2(m n-1+p)$, where $p$ is the genus of $c$ and $c^{\prime}$. For the sake of simplicity $c$ is supposed to have no cusps and to contain none of the base points. Projecting $c^{\prime}$ from the multiple point of the surface, which we have seen is of order $n-1$ for the surface and of order $m(n-1)$ for $c^{\prime}$, we find that the developable has there a multiple point of order $2 m(n-1)$. The developable intersects the surface in the 
cuspidal edge counted twice, and in a residual curve whose image is the locus sought. To find the order of the latter we have only to note that to a line of the plane corresponds on the surface a plane $n$-ic which has an $(n-1)$-fold point at the multiple point of the surface. Multiplying the order of the developable by $n$, and subtracting the $2 m(n-1)^{2}$ intersections at the multiple point and the $2 m$ on the cuspidal edge, we find that the order of the locus sought is $2(2 m n-2 m-n+n p)$. This holds for $m \geqq 2$. The locus passes $2(m+p-1)$ times through each of the points $A$. If the points $A$ were in general position, the order $r$ of the developable would remain the same; but the order of the locus would be $r n-2 m$, that is, greater by $2 m(n-1)^{2}$, and it would pass $2 m(n-1)$ more times through each of the points $A$. In addition to the singularities at the base points the locus has a number of nodes corresponding to those intersections of the double curve of the developable with the rational surface that do not occur on the cuspidal edge. Since the genus of a plane section of the developable is the same as that of the cuspidal edge, the order of the double curve is $(1 / 2)(r-1)(r-2)-m n-p$. If the cuspidal edge has a multiple point of order $s$, the double curve has there a multiple point of order $2 s(s-1)$. Therefore, the double curve of the developable in question has a multiple point of order $2 m(n-1)[m(n-1)-1]$ at the multiple point of the rational surface, and a multiple point of order 4 at each of the $(1 / 2)(m-1)(m-2)-p$ nodes of $c^{\prime}$. The number $\alpha$ of stationary planes of the cuspidal edge is given by $3(r-2)$ $-2 m n+6 p$. The point of contact of such a plane is a simple point on both the double curve and the cuspidal edge. A point where the cuspidal edge is met by a tangent to itself is a cusp on the double curve. A curve in 3-space of order $\mu$ and genus $p$ without nodes or cusps has $2(\mu-2)(\mu-3)+2 p(\mu-6)$ tangents that meet it again. This number is reduced by 4 when an apparent double point is replaced by a node, and by the order $\mu$ if the apparent double point is replaced by a cusp. In the present instance the order of the cuspidal edge is $m n$, the genus is $p$, and there is a multiple point of order $m(n-1)$ and $(1 / 2)(m-1)(m-2)-p$ nodes. The number $\gamma$ of tangents to the cuspidal edge that meet it again is thus found to be $4 m(1+m n-m-2 n)+8+2 p(m n-4)$. The number of intersections of the double curve of the developable with the ra- 
tional surface that do not take place on the cuspidal edge is $n x-2 m(n-1)^{2}[m(n-1)-1]-\alpha-2 \gamma-4[(1 / 2)(m-1)(m-2)-p]$, where $x$ is the order of the double curve given above. Each such intersection corresponds to a node on the locus sought. Therefore, considering this number of nodes and the singularities at the base points, the genus of the locus is found to be $(n-3)(3 m n-2 m-n-3)+p\left(n^{2}-8\right)$. If the base points were in general position, the corresponding expression would be as follows:* $(n-3)\left(5 m n^{2}-6 m-5 n\right) / 2+p\left(5 n^{2}-15 n+2\right) / 2$. As is to be expected, both reduce to $p$ when $n=3$. For we should then be dealing with a pencil of cubics having six fixed base points while $Q, P$, the seventh and eighth, are consecutive on the curve of order $m$. Hence to each point of this curve corresponds one point on the locus in question. And the genus of the latter we should expect to be the same whether the six fixed points are on a conic or not.

Finally if $m=1$, that is, if the two consecutive points $P, Q$ describe a line $l$, the locus of the others is that same line, and the above result does not hold. But since to $l$ there corresponds on the surface a rational plane $n$-ic having an $(n-1)$-fold point, it is seen immediately that the number of positions of $P$ on $l$ such that one of the corresponding points coincides with $P$ is the number of inflections of such an $n$-ic, that is, $3(n-2)$. And the number of positions of $P$ such that two corresponding points coincide is twice the number of double tangents, that is, $4(n-2)(n-3)$.

The University of California

* See the paper mentioned above. 